\title{
Mid- and Long-Wave Infrared Optoelectronics via Intraband Transitions in PbS Colloidal Quantum Dots
}

\author{
Iñigo Ramiro, Onur Özdemir, Sotirios Christodoulou, Shuchi Gupta, Mariona Dalmases, Iacopo Torre,
} and Gerasimos Konstantatos*

Cite This: Nano Lett. 2020, 20, 1003-1008

Read Online

ABSTRACT: Optical sensing in the mid- and long-wave infrared (MWIR, LWIR) is of paramount importance for a large spectrum of applications including environmental monitoring, gas sensing, hazard detection, food and product manufacturing inspection, and so forth. Yet, such applications to date are served by costly and complex epitaxially grown $\mathrm{HgCdTe}$ quantum-well and quantum-dot infrared photodetectors. The possibility of exploiting low-energy intraband transitions make colloidal quantum dots (CQD) an attractive lowcost alternative to expensive low bandgap materials for infrared applications. Unfortunately, fabrication of quantum dots exhibiting intraband absorption is technologically constrained by the requirement of controlled heavy doping, which has limited, so far, MWIR

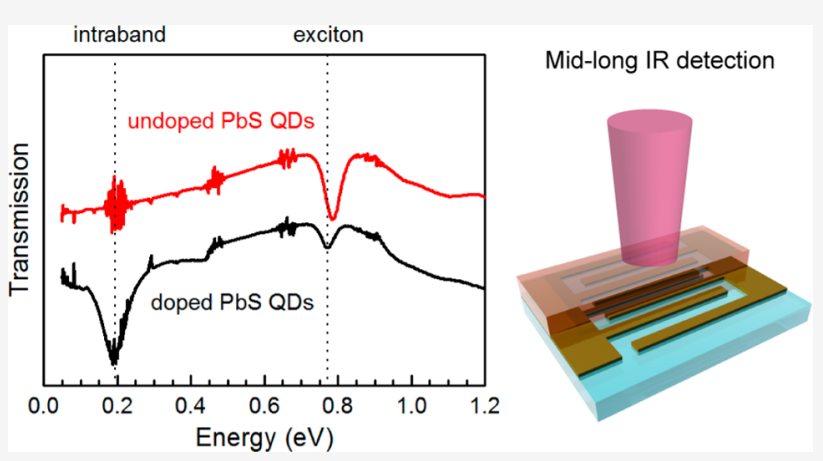
and LWIR CQD detectors to mercury-based materials. Here, we demonstrate intraband absorption and photodetection in heavily doped $\mathrm{PbS}$ colloidal quantum dots in the 5-9 $\mu \mathrm{m}$ range, beyond the $\mathrm{PbS}$ bulk band gap, with responsivities on the order of $10^{-4} \mathrm{~A} /$ $\mathrm{W}$ at $80 \mathrm{~K}$. We have further developed a model based on quantum transport equations to understand the impact of electron population of the conduction band in the performance of intraband photodetectors and offer guidelines toward further performance improvement.

KEYWORDS: Intraband absorption, lead sulfide, colloidal quantum dots, infrared photodetector, doping

C olloidal quantum dot (CQD) technology enables lowcost, flexible, and complementary metal-oxide-semiconductor (CMOS) compatible optoelectronics with spectral coverage tunable through the quantum confinement effect. These features have motivated extensive research toward CQD light-emitting diodes, ${ }^{1}$ solar cells, ${ }^{2}$ photodetectors, ${ }^{3}$ and field effect transistors. ${ }^{4}$ There is, however, a fundamental limitation in the band gap engineering of CQDs. The spectral coverage that can be reached by a CQD material is determined by the bulk band gap of the semiconductor compound. Quantum confinement can only increase the optical band gap of CQD semiconductor from a minimum value, given by the band gap of the respective bulk material. This condition restricts the number of potential applications for many CQD materials to the visible (vis), near-infrared (NIR), and short-wavelength infrared (SWIR) range of the electromagnetic spectrum. Thus, only semimetallic bulk materials or those with a very low bandgap $(<0.4 \mathrm{eV})$ can potentially give rise to CQD optoelectronics in the mid- and long-wave infrared (MWIR and LWIR). A plausible way to bypass this limit is by leveraging intraband transitions in CQDs. ${ }^{5,6}$ Intraband absorption within the conduction band (CB) can occur when the confined ground state, $1 S_{e}$, is populated with electrons. ${ }^{7,8}$ In this situation, electrons can be excited to higher energy states via absorption of photons with energy lower than the band gap. Analogous description holds for holes occupying the confined ground state of the valence band $(\mathrm{VB}), 1 \mathrm{~S}_{\mathrm{h}}$.

CQDs exhibiting intraband optical coupling (absorption ${ }^{9}$ and luminescence $\left.{ }^{10}\right)$ can be a potential alternative to current high-cost MWIR/LWIR photodetectors and quantum cascade laser technologies based on epitaxially grown $\mathrm{HgCdTe}$ or IIIV superlattices. ${ }^{11,12}$ Stable heavy doping of CQDs is required to achieve steady-state intraband absorption. ${ }^{6}$ This has been demonstrated in different materials using different doping methods (see ref 6 for a review). In particular, several groups have reported steady-state intraband absorption in lead chalcogenide (PbS, PbSe) CQDs in solution. ${ }^{13-16}$ However, none of these approaches led to stable heavily doped solidstate films, and the report of $\mathrm{Pb}$-chalcogenide CQDs for intraband optoelectronics has remained elusive. The first devices exploiting intraband transitions (MWIR photodetectors) have only recently been demonstrated using mercury

Received: October 7, 2019

Revised: January 6, 2020

Published: January 14, 2020 
(a)

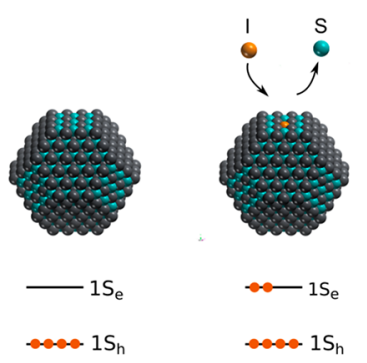

(c)

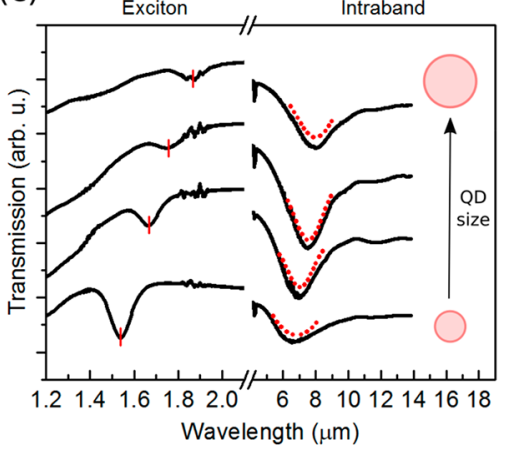

(b)

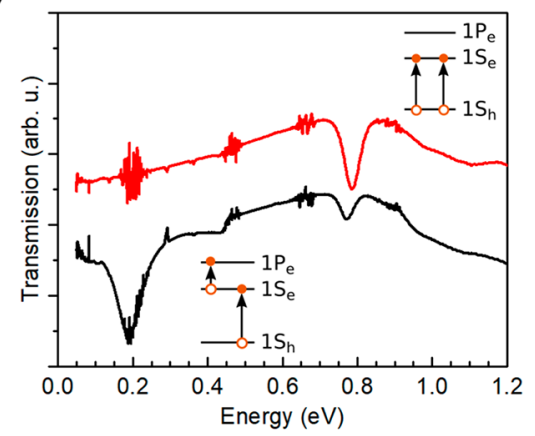

(d)

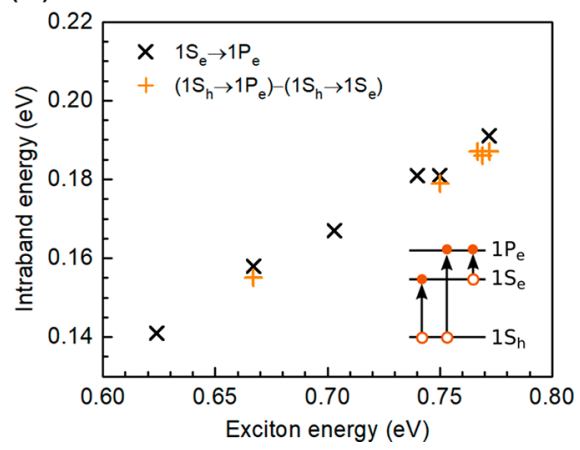

Figure 1. (a) (left) As-synthesized QDs have intrinsic low doping and exhibit filled $1 \mathrm{~S}_{\mathrm{h}}$ and empty $1 \mathrm{~S}_{\mathrm{e}}$. (right) Substitution of exposed sulfur atoms by iodine atoms leads to heavy n-type doping and a partially populated $1 \mathrm{~S}_{\mathrm{e}}$. (b) Transmission spectra of undoped (red) and doped (black) PbS CQD films. Spectra have been offset vertically for clarity. The insets illustrate interband $\left(1 S_{h} \rightarrow 1 S_{e}\right)$ and intraband $\left(1 S_{e} \rightarrow 1 P_{e}\right)$ absorption processes. (c) Transmission spectra of films of heavily doped PbS CQD with diameters ranging from $5.8 \mathrm{~nm}$ (bottom) to $8.0 \mathrm{~nm}$ (top). The central energy of the excitonic absorption for each QD size is indicated with a vertical red line. Red dotted Gaussian lines are guides to the eye representing the intraband absorption peak. (d) Intraband energy as a function of exciton energy in heavily doped PbS CQDs. Black crosses represent direct measurements of intraband transitions. Orange plus signs represent intraband energies obtained by measuring and subtracting the 2 nd and 1st excitonic peaks.

chalcogenide nanocrystals. ${ }^{9,17,18} \mathrm{HgTe}$ and $\mathrm{HgSe}$ being semimetals in their bulk form, allow access to the MWIR and LWIR through interband transitions in Hg-based CQDs. ${ }^{19}$ In this work, we demonstrate intraband absorption in $\mathrm{PbS}$ CQDs for photon energies down to $0.14 \mathrm{eV}$, well below the bulk bandgap of $\mathrm{PbS}(0.41 \mathrm{eV})$. In addition, we demonstrate photodetection in the 5-9 $\mu \mathrm{m}$ range, proving that this absorption process can be exploited in optoelectronic devices. Finally, we develop a quantum-transport model in order to evaluate the impact of the occupation of the $1 S_{e}$ state in the potential performance of intraband photodetectors.

We have achieved recently a method that achieves robust heavy doping in $\mathrm{PbS}$ CQDs, ${ }^{20}$ based on sulfur substitution by iodine via a simple ligand exchange procedure followed by infilling and capping via atomic layer deposition the CQD film with alumina. As sketched in Figure 1a, as-synthesized $\mathrm{PbS}$ CQDs exhibit low doping, and thus $1 S_{h}$ is almost completely filled and $1 S_{\mathrm{e}}$ is almost completely empty. Upon sulfur substitution by iodine, $\mathrm{PbS}$ dots are heavily doped n-type (see Figure $S 1$ ) and $1 S_{e}$ is partially occupied. In ref 20, it was found that doping is more effective with increasing dot size because larger dots contain more exposed sulfur atoms in (100) facets. For dots with a diameter smaller than $4 \mathrm{~nm}, 1 S_{\mathrm{e}}$ is almost empty. For larger dots, with diameter between $4 \mathrm{~nm}$ (exciton $\sim 1150 \mathrm{~nm}$ ) and $8 \mathrm{~nm}$ (exciton $\sim 1850 \mathrm{~nm}$ ), heavy doping is achieved and $1 S_{\mathrm{e}}$ is partially populated. For dots with diameter larger than $8 \mathrm{~nm}, 1 \mathrm{~S}_{\mathrm{e}}$ is nearly filled with $\sim 8$ electrons per $\mathrm{QD}$, corresponding to the 8 -fold degeneracy of $\mathrm{PbS} \mathrm{QDs}^{21}$
In heavily doped QDs, filling of the CB results in bleaching of interband absorption. As a counterpart, intraband absorption is then possible. ${ }^{7,22}$ To demonstrate intraband absorption in PbS CQDs, we have synthesized QDs following reported methods and fabricated films (under ambient atmosphere) with an optimized procedure for exchanging the original oleate ligands with iodine (see Methods in Supporting Information). We have prepared films of doped and undoped $\mathrm{PbS}$ CQDs of different sizes for absorption measurements. It should be reminded here that with the employed method, heavy doping is only attained after infiltration and encapsulation of the iodine-exchanged film with alumina, because this removes oxygen adsorbates, present in the samples exposed to the atmosphere, that induce p-type doping. ${ }^{20}$ Therefore, hereafter, we will consider undoped films as those in which the original oleate ligands have been exchanged with iodine but are not encapsulated and doped films as those that have been adequately encapsulated with alumina.

Figure $1 \mathrm{~b}$ shows transmission measurements of two iodineexchanged PbS QD samples, one is heavily doped while the other is not. The undoped sample exhibits strong absorption at the interband $\left(1 S_{\mathrm{h}} \rightarrow 1 S_{\mathrm{e}}\right)$ transition, as illustrated in the topright inset. In the doped sample, the exciton peak $(\sim 0.786 \mathrm{eV})$ is partially bleached and a strong intraband absorption peak $(\sim 0.191 \mathrm{eV})$ appears because of the partial population of $1 S_{e}$, as illustrated in the bottom-left inset. Transmission measurements performed on $\mathrm{PbS}$ films exchanged with thiol ligands, instead of iodine, and encapsulated with alumina do not show 
(a)

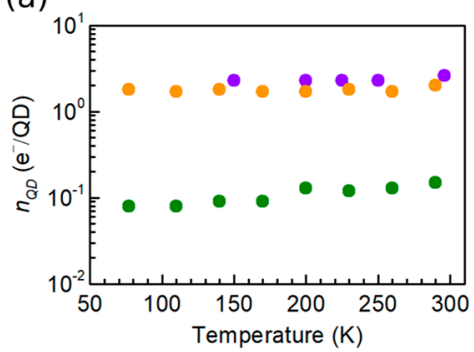

(b)
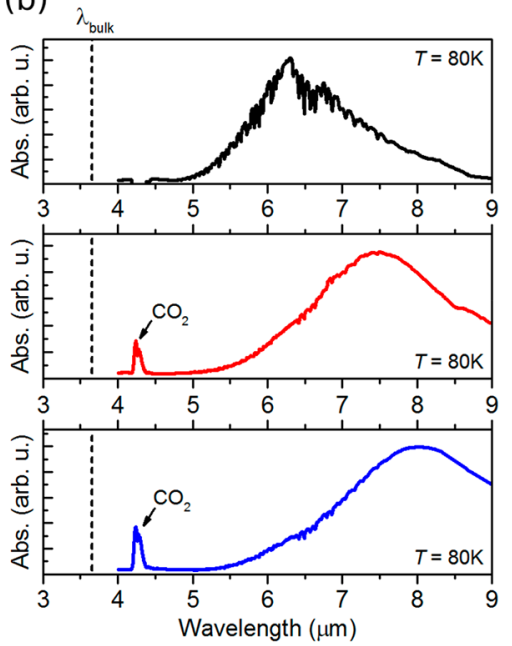

(c)

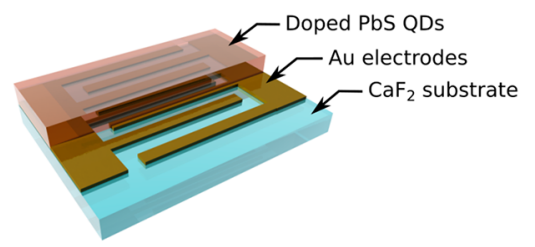

(d)
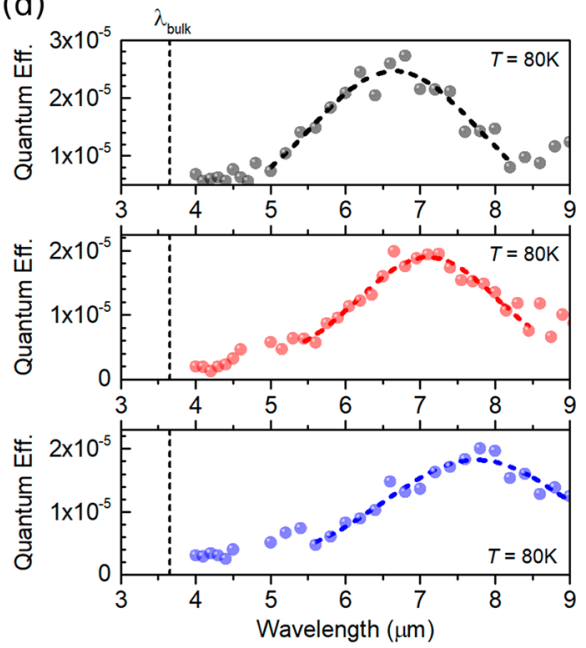

Figure 2. (a) Effect of temperature on the doping level of PbS CQD films for three different sizes of QDs. (b) Absorption spectra, measured at 80 $\mathrm{K}$, of three doped PbS CQD films with PbS of different sizes. (c) Sketch of the interdigitated devices used for photoconductivity measurements. (d) Photodetection quantum efficiency of devices using the same dots as in (c). The applied biases, $V_{\text {bias, }}$ were 1,5 and $1.6 \mathrm{~V}$, from top to bottom. The chopping frequency of the excitation light, $f_{\mathcal{c}}$, was $11 \mathrm{~Hz}$ in all cases. Gaussian fittings (dashed) are superimposed to the measured data. Vertical dashed lines indicate the band gap wavelength of bulk $\mathrm{PbS}, \lambda_{\text {bulk, }}$ at $80 \mathrm{~K}$.

exciton bleach or intraband absorption (Figure S2), further supporting the dopant role of iodine demonstrated in ref 20.

Intraband transition redshifts with increasing dot size, resulting from a progressive relaxation in the charge confinement (Figure 1c). By tuning the size of the dots, we demonstrate intraband absorption in the 6-9 $\mu \mathrm{m}$ range, as indicated by Gaussian fittings (red dotted lines). Beyond 10 $\mu \mathrm{m}$, alumina has some contribution to the absorption (see Figure S3). Note as well the progressive disappearance of the interband peak (left side of the panel) as the dot size increases, resulting from an increasing population of the $\mathrm{CB}$.

Figure 1d shows the intraband transition energy of doped $\mathrm{PbS}$ CQDs versus their exciton energy. The intraband transitions measured span from 0.14 to $0.20 \mathrm{eV}$. These energies have been obtained from absorption measurements in two different ways, as sketched in the bottom-right inset: (I) fitting direct intraband $\left(1 \mathrm{~S}_{\mathrm{e}} \rightarrow 1 \mathrm{P}_{\mathrm{e}}\right)$ transitions (black crosses); and (II) by fitting and subtracting the $1 \mathrm{~S}_{\mathrm{h}} \rightarrow 1 \mathrm{P}_{\mathrm{e}}$ and $1 \mathrm{~S}_{\mathrm{h}} \rightarrow$ $1 S_{\mathrm{e}}$ transition energies (orange plus signs). The latter method is supported by the fact that parity selection rules are relaxed in $\mathrm{PbS}$ QDs, allowing the otherwise forbidden $1 \mathrm{~S}_{\mathrm{h}} \rightarrow 1 \mathrm{P}_{\mathrm{e}}$ transition. $^{23,24}$ Both methods give similar results, thus confirming the intraband nature of the low-energy absorption of our QDs. Examples of the fits are shown in Figure S4.

Our doping method was previously proven to be robust with time (>2 months). ${ }^{20}$ Robustness with temperature is also of paramount importance for optoelectronic applications in the mid- and long-wave infrared. In particular, some applications such as infrared photodetection require low temperatures to achieve the highest performance. ${ }^{11}$ We define $n_{\mathrm{QD}}$ as the doping level of the CQD film, measured in electrons per QD $\left(\mathrm{e}^{-} / \mathrm{QD}\right)$. Figure $2 \mathrm{a}$ shows the dependence of the doping level with temperature in two $\mathrm{PbS}$ QDs samples. The doping level was obtained from field effect transistor (FET) measurements (see Figure S5), following the method described in ref 20. Figure $2 \mathrm{a}$ demonstrates that the efficacy of the employed doping method is weakly dependent on temperature in the $80-300 \mathrm{~K}$ range. Consequently, intraband absorption is still accessible at low temperatures (see Figure $2 \mathrm{~b}$ and Figure S6) enabling therefore the development of intraband $\mathrm{PbS}$ CQD photodetectors. We have thus fabricated interdigitatedelectrode devices (see Figure 2c) for photoconductivity measurements. Devices were fabricated on transparent $\mathrm{CaF}_{2}$ substrates to avoid sample heating due to parasitic absorption in the substrate. Figure $2 \mathrm{~d}$ plots the intraband quantum efficiency, QE, spectra of devices measured under monochromatic light at $80 \mathrm{~K}$. The vertical dashed lines indicate the bandgap of bulk $\mathrm{PbS}$ at $80 \mathrm{~K}(\sim 3.7 \mu \mathrm{m}) .{ }^{25}$ Our measurements demonstrate intraband photodetection in the 5-9 $\mu \mathrm{m}$ range (MWIR and LWIR), thus breaking the limit set by the band gap value of the bulk in CQD optoelectronics. Beyond $9 \mu \mathrm{m}$, the light power density reaching the sample was too low to produce a measurable signal.

Measurements of the dependence of the photocurrent with the modulation frequency (see Figure S7) indicate that both intraband and interband detection are slow: $>30$ and $>200 \mathrm{~ms}$, respectively. We have measured the specific detectivity, $D^{*}$, of our devices (see Methods in Supporting Information). At $80 \mathrm{~K}$ 
and $11 \mathrm{~Hz}$, we have obtained peak responsivities and detectivities of $1.5 \times 10^{-4} \mathrm{AW}^{-1}$ and $4 \times 10^{4}$ Jones at 6.8 $\mu \mathrm{m} ; 1.1 \times 10^{-4} \mathrm{AW}^{-1}$ and $8 \times 10^{4}$ Jones at $7.3 \mu \mathrm{m}$; and $1.3 \times$ $10^{-4} \mathrm{AW}^{-1}$ and $4 \times 10^{4}$ Jones at $8.0 \mu \mathrm{m}$, for top, middle, and bottom panels of Figure $2 \mathrm{~d}$, respectively. Correcting by the shadowing factor of the interdigitated electrodes $(\sim 50 \%)$ and the reflectivity of the $\mathrm{PbS}$ layer would give rise to internal detectivity values on the order of $10^{5}$ Jones. While our detectors yield similar responsivity values with the first reported $\mathrm{HgSe}$ CQD intraband photodetectors, ${ }^{9}$ the detectivity of our devices is currently inferior to state-of-the-art mercury-based devices. ${ }^{26}$ The performance of our devices is largely limited by high noise due to the large dark current. The high doping levels of our CQD films enable intraband absorption but at the same time result in high conductivities (see Figure S5 and ref 20) and hence dark currents. Therefore, it would be useful to evaluate the impact of electron population on the performance of our PbS CQD photodetectors.

The occupancy factor of $1 S_{\mathrm{e}}$ has a strong impact in the conductance of CQD films. ${ }^{27,28}$ Reference 29 analyzes the performance of quantum-dot infrared photodetectors (QDIP) as a function of the doping level, suggesting that minimum dark current and best performance occur for a doping level equal to the degeneracy of $1 S_{e}$ (included spin). This work considers QDs epitaxially grown within a matrix of higher bandgap, which acts as a barrier. In that case, transport only occurs through the barrier and not through $1 S_{\mathrm{e}}$ states. In a CQD film, however, transport between dots can take place through $1 \mathrm{~S}_{\mathrm{e}}$ as well as, in principle, through $1 \mathrm{P}_{\mathrm{e}}$, as sketched in Figure 3a. To shed further insights on the performance potential of intraband CQDs photodetectors, we have developed a simple transport model for doped CQD films, based on quantum transport equations. The model does not aim to obtain quantitative values of performance but rather to qualitatively assess the role of doping on some basic photodetector figures of merit. It is noteworthy that at this level the effects of doping on the lifetime of carriers in higher excited states as well as potential secondary effects on the mobility via carrier-carrier scattering are not taken into account. It evaluates, as a function of $n_{\mathrm{QD}}$, (I) the steady-state conductance under a given applied bias prior to illumination, $\bar{G}$; and (II) the change in conductance, $\Delta G$, caused by intraband absorption in the QDs. The ratio $\Delta G / \bar{G}$ will provide a qualitative indication of the potential detectivity of our devices, since detectivity is proportional to $\Delta G$ and inversely proportional to the noise, which in turn increases with $\bar{G}$. The starting point of our model is the linear response formula of conductivity $(G)$ at finite temperatures ${ }^{30}$ given by eq 1

$$
G=\frac{2 e^{2}}{h} \int \overline{\mathcal{F}}(E)\left(-\frac{\partial f}{\partial E}\right) \mathrm{d} E
$$

where $\frac{2 e^{2}}{h}$ is the conductance quantum, $e$ is the elementary charge, $h$ is Planck's constant, $\overline{\mathcal{F}}$ is the product of the number of propagating modes, and the electron transmission probability per mode

$$
f(E)=\frac{1}{e^{\left(\frac{E-E_{F}}{k_{\mathrm{B}} T}\right)}+1}
$$

is the Fermi function and determines the electron occupancy factor (from 0 to 1 ) at levels of energy $E, E_{\mathrm{F}}$ is the Fermi energy, and $k_{\mathrm{B}}$ is Boltzmann constant. The details and (a)

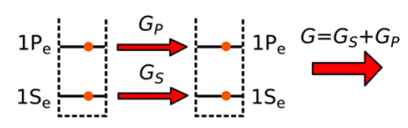

(b)

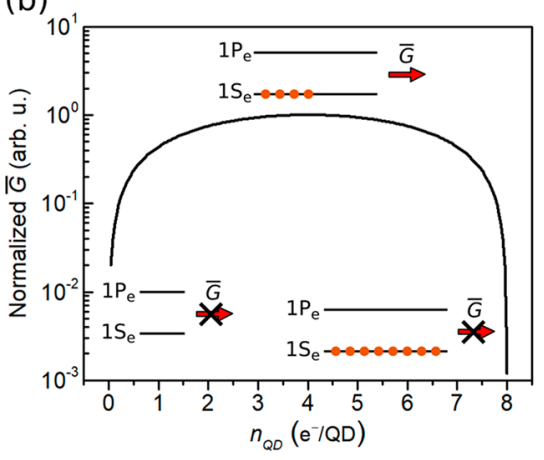

(c)

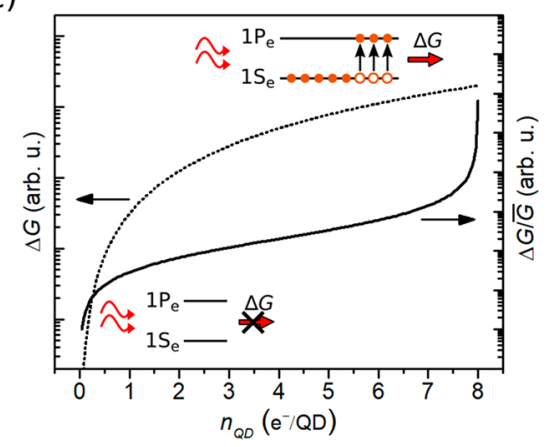

Figure 3. (a) Scheme of the QD transport model for two adjacent QDs. $G, G_{S}$, and $G_{\mathrm{P}}$ are total conductance, conductance through $1 S_{\text {e }}$ channels, and conductance through $1 \mathrm{P}_{\mathrm{e}}$ channels, respectively. (b) Modeled normalized steady-state conductance, $\bar{G}$, of a PbS CQD film as a function of the doping level $n_{\mathrm{QD}}$. (c) Left axis, broken line: modeled increase in conductance, $\Delta G$, due to intraband light absorption. Right axis, solid line: modeled photodetection performance, expressed as $\Delta$, as a function of $n_{\mathrm{QD}}$. The insets in $(\mathrm{b}, \mathrm{c})$ illustrate the impact of the doping in the population of $1 S_{e}$ and, therefore, in the value of $\bar{G}$ and $\Delta$.

development of the model are included in the Supporting Information. The main result of the model is eq 3 :

$$
\frac{\Delta G}{\bar{G}}=\frac{a\left(\frac{8}{n_{Q D}}\right)+b}{1-\left(\frac{8}{n_{Q D}}\right)}
$$

where 8 represents the degeneracy of $1 S_{e}$, and $a$ and $b$ are parameters that depend on the intensity of the infrared illumination and the degeneracy of $1 S_{e}$ and $1 P_{e}$. Equation 3 indicates that the highest possible occupation of $1 \mathrm{~S}_{\mathrm{e}}$ is required in order to achieve high detectivity, as it is the case for QDIPs. This is so because noise (related to $\bar{G}$ ) decreases and responsivity (related to $\Delta G$ ) increases for high values of $n_{\mathrm{QD}}$, as illustrated in Figure 3b,c. Our results suggest that the low detectivity measured in our devices can be largely due to the fact that $1 S_{\mathrm{e}}$ is not completely filled, therefore pointing toward further research to maximize the doping density closer to the state degeneracy value.

In summary, we have demonstrated intraband absorption and photodetection in robust heavily doped $\mathrm{PbS} C \mathrm{CQD}$ solid state films. Because of the relative large bandgap of the $\mathrm{PbS}$ 
bulk crystal, intraband absorption in PbS CQDs can access ranges of the spectrum that are unattainable by interband transitions. This bandgap modulation strategy further expands the solution-processed material availability toward the MWIR and LWIR for sensing and light emission applications. The size-tunable spectral line width of intraband transitions employed here taken together with the facile integration of CQDs of different sizes may lead to CMOS compatible lowcost multispectral imaging systems in the infrared.

During the revision process of the present manuscript, Law et al. have demonstrated the rise of intraband absorption in $\mathrm{PbSe} \mathrm{QD}$ superlattices following halide exchange and $\mathrm{Al}_{2} \mathrm{O}_{3}$ infilling with $\mathrm{ALD},{ }^{31}$ corroborating the findings of our work and demonstrating the applicability of this process to lead chalcogenide QDs. The efficacy of the doping method employed herein is limited by the amount of exposed sulfur, imposed by the size of the nanocrystal. It would be desirable to circumvent this limitation so that intraband absorption can be enabled even in smaller dots. To this end, we speculate that a postsynthesis sulfur (or another chalcogen) treatment could increase the amount of anions available for substitution even at smaller QDs. Alternatively, the use of halide ions in situ during the growth of QDs could potentially circumvent the need for exposed 001 facets for the substitution to take place.

\section{ASSOCIATED CONTENT}

\section{SI Supporting Information}

The Supporting Information is available free of charge at https://pubs.acs.org/doi/10.1021/acs.nanolett.9b04130.

Methods, absorption measurements, FET measurements, intraband photocurrent at different excitation frequencies, and transport model (PDF)

\section{AUTHOR INFORMATION}

\section{Corresponding Author}

Gerasimos Konstantatos - ICFO-Institut de Ciencies

Fotòniques, Castelldefels, Barcelona, Spain, and ICREA-

Institució Catalana de Recerca i Estudis Avançats,

Barcelona, Spain; (1) orcid.org/0000-0001-7701-8127;

Email: Gerasimos.Konstantatos@icfo.eu

\section{Other Authors}

Iñigo Ramiro - ICFO-Institut de Ciencies Fotòniques, Castelldefels, Barcelona, Spain; (1) orcid.org/0000-00029663-4002

Onur Özdemir - ICFO-Institut de Ciencies Fotòniques, Castelldefels, Barcelona, Spain; (1) orcid.org/0000-00018692-5087

Sotirios Christodoulou - ICFO-Institut de Ciencies Fotoniques, Castelldefels, Barcelona, Spain; (1) orcid.org/ 0000-0001-7020-3661

Shuchi Gupta - ICFO-Institut de Ciencies Fotòniques, Castelldefels, Barcelona, Spain

Mariona Dalmases - ICFO-Institut de Ciencies

Fotòniques, Castelldefels, Barcelona, Spain

Iacopo Torre - ICFO-Institut de Ciencies Fotòniques, Castelldefels, Barcelona, Spain

Complete contact information is available at: https://pubs.acs.org/10.1021/acs.nanolett.9b04130

\section{Notes}

The authors declare no competing financial interest.

\section{ACKNOWLEDGMENTS}

I.R. acknowledges support from the Ministerio de Economía, Industria y Competitividad of Spain via a Juan de la Cierva fellowship. The authors acknowledge financial support from the European Research Council (ERC) under the European Union's Horizon 2020 research and innovation programme (Grant Agreement 725165), the Spanish Ministry of Economy and Competitiveness (MINECO), and the "Fondo Europeo de Desarrollo Regional” (FEDER) through Grant TEC201788655-R. The authors also acknowledge financial support from Fundacio Privada Cellex, the program CERCA and from the Spanish Ministry of Economy and Competitiveness, through the "Severo Ochoa" Programme for Centres of Excellence in R\&D (SEV-2015-0522). S.C. acknowledge support from a Marie Curie Standard European Fellowship ("NAROBAND", H2020-MSCA-IF-2016-750600).

\section{REFERENCES}

(1) Shirasaki, Y.; Supran, G. J.; Bawendi, M. G.; Bulović, V. Emergence of Colloidal Quantum-Dot Light-Emitting Technologies. Nat. Photonics 2013, 7 (1), 13-23.

(2) Carey, G. H.; Abdelhady, A. L.; Ning, Z.; Thon, S. M.; Bakr, O. M.; Sargent, E. H. Colloidal Quantum Dot Solar Cells. Chem. Rev. 2015, 115 (23), 12732-12763.

(3) Konstantatos, G.; Sargent, E. H. Colloidal Quantum Dot Photodetectors. Infrared Phys. Technol. 2011, 54 (3), 278-282.

(4) Hetsch, F.; Zhao, N.; Kershaw, S. V.; Rogach, A. L. Quantum Dot Field Effect Transistors. Mater. Today 2013, 16 (9), 312-325.

(5) Jagtap, A.; Livache, C.; Martinez, B.; Qu, J.; Chu, A.; Gréboval, C.; Goubet, N.; Lhuillier, E. Emergence of Intraband Transitions in Colloidal Nanocrystals [Invited]. Opt. Mater. Express 2018, 8 (5), 1174.

(6) Kim, J.; Choi, D.; Jeong, K. S. Self-Doped Colloidal Semiconductor Nanocrystals with Intraband Transitions in Steady State. Chem. Commun. 2018, 54 (61), 8435-8445.

(7) Shim, M.; Guyot-Sionnest, P. N-Type Colloidal Semiconductor Nanocrystals. Nature 2000, 407 (6807), 981-983.

(8) Wang, C.; Shim, M.; Guyot-Sionnest, P. Electrochromic Nanocrystal Quantum Dots. Science (Washington, DC, U. S.) 2001, 291 (5512), 2390-2392.

(9) Deng, Z.; Jeong, K. S.; Guyot-Sionnest, P. Colloidal Quantum Dots Intraband Photodetectors. ACS Nano 2014, 8 (11), 1170711714.

(10) Deng, Z.; Guyot-Sionnest, P. Intraband Luminescence from $\mathrm{HgSe} / \mathrm{CdS}$ Core/Shell Quantum Dots. ACS Nano 2016, 10 (2), 2121-2127.

(11) Martyniuk, P.; Rogalski, A. Quantum-Dot Infrared Photodetectors: Status and Outlook. Prog. Quantum Electron. 2008, 32 (3), $89-120$.

(12) Gmachl, C.; Capasso, F.; Sivco, D. L.; Cho, A. Y. Recent Progress in Quantum Cascade Lasers and Applications. Rep. Prog. Phys. 2001, 64 (11), 1533-1601.

(13) Koh, W. K.; Koposov, A. Y.; Stewart, J. T.; Pal, B. N.; Robel, I.; Pietryga, J. M.; Klimov, V. I. Heavily Doped N-Type PbSe and PbS Nanocrystals Using Ground-State Charge Transfer from Cobaltocene. Sci. Rep. 2013, 3 (1), 2004.

(14) Lu, H.; Carroll, G. M.; Chen, X.; Amarasinghe, D. K.; Neale, N. R.; Miller, E. M.; Sercel, P. C.; Rabuffetti, F. A.; Efros, A. L.; Beard, M. C. N -Type PbSe Quantum Dots via Post-Synthetic Indium Doping. J. Am. Chem. Soc. 2018, 140 (42), 13753-13763.

(15) Kroupa, D. M.; Hughes, B. K.; Miller, E. M.; Moore, D. T.; Anderson, N. C.; Chernomordik, B. D.; Nozik, A. J.; Beard, M. C. Synthesis and Spectroscopy of Silver-Doped PbSe Quantum Dots. J. Am. Chem. Soc. 2017, 139 (30), 10382-10394. 
(16) Araujo, J. J.; Brozek, C. K.; Kroupa, D. M.; Gamelin, D. R. Degenerately N-Doped Colloidal PbSe Quantum Dots: Band Assignments and Electrostatic Effects. Nano Lett. 2018, 18 (6), 3893-3900.

(17) Goubet, N.; Livache, C.; Martinez, B.; Xu, X. Z.; Ithurria, S.; Royer, S.; Cruguel, H.; Patriarche, G.; Ouerghi, A.; Silly, M.; et al. Wave-Function Engineering in $\mathrm{HgSe} / \mathrm{HgTe}$ Colloidal Heterostructures to Enhance Mid-Infrared Photoconductive Properties. Nano Lett. 2018, 18 (7), 4590-4597.

(18) Lhuillier, E.; Scarafagio, M.; Hease, P.; Nadal, B.; Aubin, H.; Xu, X. Z.; Lequeux, N.; Patriarche, G.; Ithurria, S.; Dubertret, B. Infrared Photodetection Based on Colloidal Quantum-Dot Films with High Mobility and Optical Absorption up to THz. Nano Lett. 2016, 16 (2), 1282-1286.

(19) Keuleyan, S. E.; Guyot-Sionnest, P.; Delerue, C.; Allan, G. Mercury Telluride Colloidal Quantum Dots: Electronic Structure, Size-Dependent Spectra, and Photocurrent Detection up to $12 \mathrm{Mm}$. ACS Nano 2014, 8 (8), 8676-8682.

(20) Christodoulou, S.; Ramiro, I.; Othonos, A.; Figueroba, A.; Dalmases, M.; Özdemir, O.; Pradhan, S.; Itskos, G.; Konstantatos, G. Single-Exciton Gain and Stimulated Emission Across the Infrared Optical Telecom Band from Robust Heavily-Doped PbS Colloidal Quantum Dots. 2019, arXiv:1908.03796 [physics.app-ph].

(21) Kang, I.; Wise, F. W. Electronic Structure and Optical Properties of $\mathrm{PbS}$ and PbSe Quantum Dots. J. Opt. Soc. Am. B 1997, 14 (7), 1632.

(22) Schimpf, A. M.; Knowles, K. E.; Carroll, G. M.; Gamelin, D. R. Electronic Doping and Redox-Potential Tuning in Colloidal Semiconductor Nanocrystals. Acc. Chem. Res. 2015, 48 (7), 1929-1937.

(23) Diaconescu, B.; Padilha, L. A.; Nagpal, P.; Swartzentruber, B. S.; Klimov, V. I. Measurement of Electronic States of PbS Nanocrystal Quantum Dots Using Scanning Tunneling Spectroscopy: The Role of Parity Selection Rules in Optical Absorption. Phys. Rev. Lett. 2013, 110 (12), 127406.

(24) Nootz, G.; Padilha, L. A.; Olszak, P. D.; Webster, S.; Hagan, D. J.; Van Stryland, E. W.; Levina, L.; Sukhovatkin, V.; Brzozowski, L.; Sargent, E. H. Role of Symmetry Breaking on the Optical Transitions in Lead-Salt Quantum Dots. Nano Lett. 2010, 10 (9), 3577-3582.

(25) Gibbs, Z. M.; Kim, H.; Wang, H.; White, R. L.; Drymiotis, F.; Kaviany, M.; Jeffrey Snyder, G. Temperature Dependent Band Gap in $\mathrm{PbX}(\mathrm{X}=$ = S, Se, Te). Appl. Phys. Lett. 2013, 103 (26), 262109.

(26) Livache, C.; Martinez, B.; Goubet, N.; Gréboval, C.; Qu, J.; Chu, A.; Royer, S.; Ithurria, S.; Silly, M. G.; Dubertret, B.; et al. A Colloidal Quantum Dot Infrared Photodetector and Its Use for Intraband Detection. Nat. Commun. 2019, 10 (1), 2125.

(27) Guyot-Sionnest, P. Electrical Transport in Colloidal Quantum Dot Films. J. Phys. Chem. Lett. 2012, 3 (9), 1169-1175.

(28) Vanmaekelbergh, D.; Liljeroth, P. Electron-Conducting Quantum Dot Solids: Novel Materials Based on Colloidal Semiconductor Nanocrystals. Chem. Soc. Rev. 2005, 34 (4), 299-312.

(29) Phillips, J. Evaluation of the Fundamental Properties of Quantum Dot Infrared Detectors. J. Appl. Phys. 2002, 91 (7), 45904594.

(30) Datta, S. Electronic Transport in Mesoscopic Systems; Cambridge University Press, 1997.

(31) Abelson, A.; Qian, C.; Salk, T.; Luan, Z.; Fu, K.; Zheng, J. G.; Wardini, J. L.; Law, M. Collective Topo-Epitaxy in the Self-Assembly of a 3D Quantum Dot Superlattice. Nat. Mater. 2020, 19, 49. 